\title{
DNA methylation profile of psoriatic skins from different body locations
}

\author{
Mingshun $\mathrm{Wu}^{1,2}$, Xueying $\mathrm{Li}^{1,2}$, Chaowen Zhang ${ }^{1,2}$, Chuanliang Zhang ${ }^{1,2}$, Danfeng Qian ${ }^{1,2}$, \\ Jie Ma ${ }^{1,2}$, Minglong Cai ${ }^{1,2}$, Lili Tang ${ }^{1,2}$, Hui Cheng ${ }^{1,2}$, Changbing Shen ${ }^{3}$, Gang Chen ${ }^{1,2}$, \\ Xiaodong Zheng ${ }^{1,2}$, Xuejun Zhang**,1,4 \& Fusheng Zhou*,1,2 \\ ${ }^{1}$ Department of Dermatology, Institute of Dermatology, the First Affiliated Hospital, Anhui Medical University, Hefei 230032, PR \\ China \\ ${ }^{2}$ The Key Laboratory of Dermatology (Anhui Medical University), Ministry of Education, Hefei 230032, PR China \\ ${ }^{3}$ Department of Dermatology, China-Japan Friendship Hospital, Beijing 100029, PR China \\ ${ }^{4}$ Department of Dermatology, Institute of Dermatology, Huashan Hospital, Fudan University, Shanghai 200040, PR China \\ *Author for correspondence: biozhoufs@163.com \\ **Author for correspondence: ayzxj@vip.sina.com
}

Aim: To understand whether the anatomical location of origin plays a role in shaping the DNA methylation (DNAm) landscape of psoriatic skins. Patients \& methods: A number of 108 psoriatic and 57 control skin samples were grouped based on their anatomical locations. Two group t-tests were used to identify those differentially methylated sites and regions. Target region methylation loci were validated by bisulfate conversion sequencing. The correlations of DNAm with pathological features, DNAm and gene expression were also interrogated. Results: Our analysis revealed 315 location-specific differentially methylated sites for back, 291 for the extremities and 801 for abdomen. Moreover, we observed that the extremity-specific loci cg21942490 located on HOXA9 is associated with hyperkeratosis. We further observed that HOXA5 and KIAA1949 are differential methylation regions. Conclusion: Our study shown evidence of anatomical location-dependent DNAm pattern in psoriasis skins, and thus provided new insights into the pathogenesis of this disease.

First draft submitted: 29 December 2018; Accepted for publication: 3 October 2019; Published online: 8 November 2019

Keywords: CSSS • different body locations $\bullet$ DMRs $\bullet$ DMSs $\bullet$ growth factor $\bullet$ HOXA5 and KIAA1949 • locationspecific sites $\bullet$ pathological features $\bullet$ psoriasis

Psoriasis is a chronic, immune-mediated skin disorder affecting 2-3\% of the world's population. It has a higher prevalence in American and Canadian populations (4.6 and 4.7\%, respectively), compared with $0.4-0.7 \%$ in African, African-American and Asian populations [1,2]. The pathogenesis of psoriasis remains incompletely understood, but there is increasing evidence indicated that aberrant DNA methylation (DNAm) patterns are one of the most likely causes, encompassing differential methylation sites (DMSs) and differential methylation regions (DMRs) [3-5].

Pathological, therapeutic and molecular variations have been noticed among psoriasis originated from different anatomical body locations, despite the relatively consistent clinical manifestation of the disease. First, the severity varies across different body locations. Specifically, in case of extremities, torsos are prone to have more severe symptoms [6,7]. Second, the location of the lesions on the body may affect the response to drugs in patients with immune diseases. There is some evidence that DMSs in psoriatic skin can revert, after phototherapy or after 1 month of anti-TNF- $\alpha$ therapy, to that observed in normal tissue. This observation might indirectly confirm the association of DNAm with the clinical outcome of interindividual locations $[4,8]$. Third, it has been previously reported that many DMRs, particular those in hypomethylated regions, have tissue-specific functions that is tightly linked to the anatomical locations that raised the disease [9-13]. Fourth, gene expression profile of psoriatic skins might be influenced by their biopsy locations [14]. These lines of findings suggest that body location may play an important role in psoriasis. 
Despite previous progresses in terms of aberrant DNAm landscape in psoriasis, the potential influence of different body locations has not been examined. Furthermore, a recent study [15] on the role of DNAm in the clinical cardinal histopathological regulation of psoriasis inspired us to explore the relationship among DNAm, histopathologies and body location. We have previously obtained genome-wide DNAm data by using HumanMethylation450 BeadChip. Here, we conducted concrete association analysis among them.

\section{Patients \& methods}

\section{Sample preparation}

We previously performed a genome-wide DNAm study which included 114 psoriatic skin and 62 normal skin samples [3]. Here we grouped these samples based on their anatomical locations and disease state. Some samples were excluded because they were not the entry objects of our research. All samples were collected from the Department of Dermatology, the First Affiliated Hospital, Anhui Medical University, China. The patients' clinical and histopathological information, including sex, age and Psoriasis Area and Severity Index (PASI) scores, was showed in Supplementary Tables 1 and 2.

\section{Identifying DMSs}

There are two types of probes with different hybridization chemistries for Illumina 450k methylation BeadChip (Illumina Inc, CA, USA). Such design of including both type-I and -II probes may pose certain bias if not well adjusted. To account for this, we first performed probe normalization by using BMIQ ( $\beta$ Mixture Quantile dilation), which is a model-based intra-array normalization strategy [16]. We then used t-test to compare the difference between groups. DMSs were considered those fulfilling the criteria of $\delta-\beta>0.1$ and Benjamini-Hochberg $(\mathrm{BH})$ corrected $\mathrm{p}<0.001$. All statistical analyses were performed using the R package ChAMP [17].

\section{Algorithm for detecting DMRs}

The Bumphunter algorithm was used to detect DMRs. This algorithm is not based on previous DMP output. Initially, all methylation probes were separated into clusters. Each cluster contained at least seven probes, and the maximum distance between two nearby probes was set to 300 base pairs (bp). Probes from different chromosomes were separated. We then fitted a statistical model to calculate the value of bumps for each probe. Consecutive probes meeting a cut-off value of 0.05 were classified into the same region.

$$
Y \mathrm{ij}=\beta 0\left(l_{j}\right)+\beta 1\left(l_{j}\right) X_{j}+\varepsilon_{\mathrm{ij}}
$$

where $i$ represents the subject, $l_{\mathrm{j}}$ represents the jth probe, $X_{\mathrm{j}}$ is the disease status. $\epsilon_{\mathrm{ij}}$ is the measurement error, $\beta O\left(l_{\mathrm{j}}\right)$ is a baseline and $\beta 1\left(l_{\mathrm{j}}\right) X_{\mathrm{j}}$ is the parameter of interest, which is a function of probes. We assume that $\beta 1(D)$ will be equal to zero over most of the genomes, and we want to identify stretched where $\beta 1(l) \neq 0$, which we call bumps. Permutations were performed to create null distributions for the candidate regions. For each observed candidate region, we determined how many null regions were 'more extreme' (longer and higher average value). The p-value was calculated as the percent of candidate regions obtained from the permutations that are as extreme as the observed region. Family-wise error rate (FWER) was the proportion of permutations that had at least one region as extreme as the observed region. We also computed the p-value and FWER for the area of the regions. The result of Bumphunter analysis contained all the DMRs detected, with their length, clusters and number of CpGs annotated.

\section{Gene Ontology \& the Kyoto Encyclopedia of Genes \& Genomes}

Gene Ontology (GO) and Kyoto Encyclopedia of Genes and Genomes (KEGG) pathways analyses were carried out for the differentially methylated genes between groups using R package cluster Profiler [18]. Gene sets that showed differential methylation in three different body locations were searched against a default Homo sapiens population background, and these result were matched with GO biological processes and KEGG pathways. Enrichment test was based on the hypergeometric distribution. The level of statistic significance was set to a Benjamini-Hochberg (BH) corrected $\mathrm{p}<0.05$. 
Figure 1. Comparison of the number of differential methylation sites identified in psoriatic skin versus normal skin samples among back, extremities and abdomen.

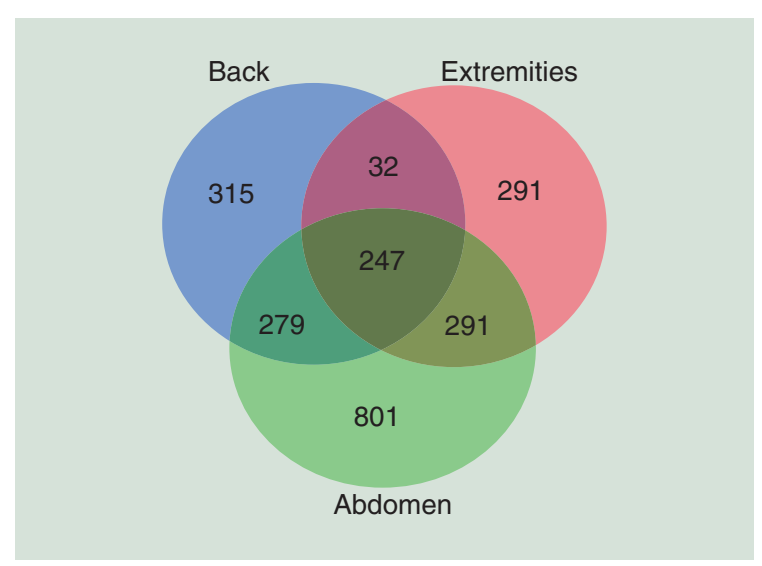

\section{Bisulfite sequencing}

The DNA methylation level was analyzed using MethylTargetTM (Genesky Biotechnologies, Inc., Shanghai, China), a next-generation sequencing (NGS) based multiple-targeted CpG methylation analysis method. Specifically, the genomic regions of interest were analyzed and transformed to bisulfite-converted sequences by the geneCpG software. PCR primer sets were designed from bisulfate-converted DNA using the Methylation Primer software.

Genomic DNA (400 ng) was subjected to sodium bisulfite treatment using EZ DNA Methylation ${ }^{T M}$-GOLD Kits (Zymo Research Corporation, CA, USA) according to the manufacturer's protocols. Libraries from different samples were quantified and pooled together, followed by sequencing on the Illumina NextSeq platform (Illumina Inc, CA, USA) according to the manufacturer's protocols. Sequencing was performed with a $2 \times 150$ bp paired-end mode. Statistical tests were performed by t-test and Analysis of Variance (ANOVA).

\section{Results}

\section{Location-specific DMSs for psoriasis}

During our genome-wide methylation analysis, we considered three pairwise comparisons: $\mathrm{PP}_{\text {back }}$ versus $\mathrm{NN}_{\text {back; }}$ $\mathrm{PP}_{\text {extremities }}$ versus $\mathrm{NN}_{\text {extremities }}$ and $\mathrm{PP}_{\text {abdomen }}$ versus $\mathrm{NN}_{\text {abdomen }}$. At a cutoff of $\mathrm{p}_{\mathrm{BH} \text {-adjusted }}<0.001$ and $\delta-\beta>0.1$, we identified 873 DMSs for the back, 861 for the extremities and 1618 for the abdomen. Among these DMSs 247 are shared across the three comparisons (Supplementary Tables 3, 4 \& 5 \& Figure 1).

For illustration, we selected two most significantly hypomethylated and two hypermethylated DMSs for each body location (Figure 2). We noticed that some of these genes are involved in immune response, one of the most fundamental processes that contributes to psoriasis development. One example is the extremity-specific site $\operatorname{cg} 23394673$ in the body of TNFRSF4 $\left(\mathrm{p}=1.88 \times 10^{-7}, \delta=0.106\right)$. TNFRSF4 was regarded as a major signal source of NF-KB involved proliferation and differentiation of keratinocytes [19,20]. Abdomen-specific hypomethylated site cg07964527 was located inside the gene body of PDE8A, implicating CD4 ${ }^{+} \mathrm{T}$ cell immunological activation processes $[21,22]$. However, another interesting loci cg22272840 was located in the gene body of $\operatorname{SOX7}(\delta=0.115$, $\left.\mathrm{p}=1.82 \times 10^{-4}\right)$, which is required for immune vascular development [23].

To further validate our findings, we selected four DMSs for each body location (12 in total) and carried out target region bisulfate conversion sequencing using $43 \mathrm{PP}$ and $47 \mathrm{NN}$ samples (back: 12 PP vs $16 \mathrm{NN}$; extremity: $14 \mathrm{PP}$ vs $13 \mathrm{NN}$; abdomen: $17 \mathrm{PP}$ vs $18 \mathrm{NN}$ ). Due to technical limitations, two DMSs failed to design target capture probes. After all, we found ten DMSs showing significant changes for the remaining loci, suggesting considerable reliability for our array results (Table $1 \&$ Supplementary Table 6).

We next performed the functional annotation for DMS-related genes. GO analysis of variably methylated genes indicated some specific features for the back, extremities and abdomen. The biological processes 'microtubule binding and cell adhesion molecule binding' were significantly enriched in samples from the back, whereas 'actin binding and transcription co-activator activity' were enriched in the extremities. Abdomen-specific GO terms included 'TGF- $\beta$ binding and phosphatidylinositol binding'. Besides location-specific GO classes, we also found the common functional terms 'growth factor binding' among the three locations, suggesting its fundamental roles for disease manifestations (Figure 3). Detailed GO results can be found in Supplementary Table 8. 

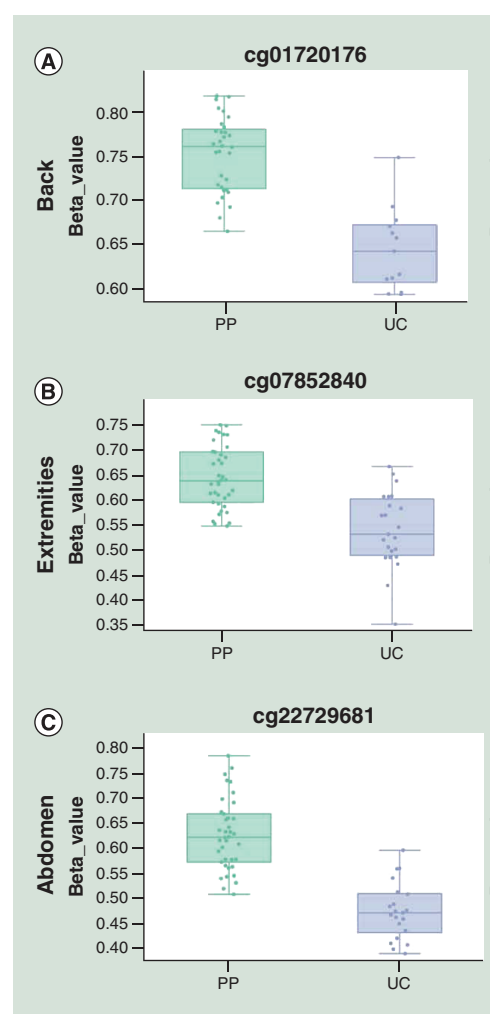
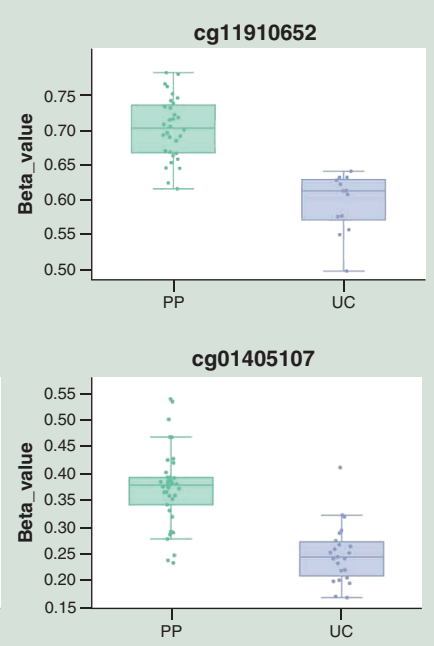

$\operatorname{cg} 01866518$

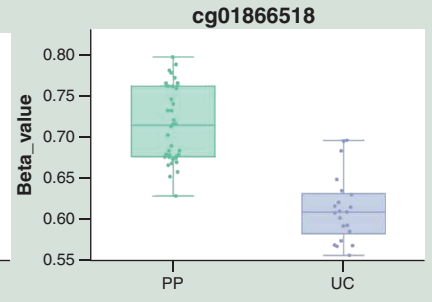

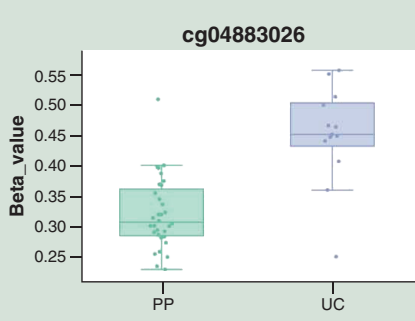

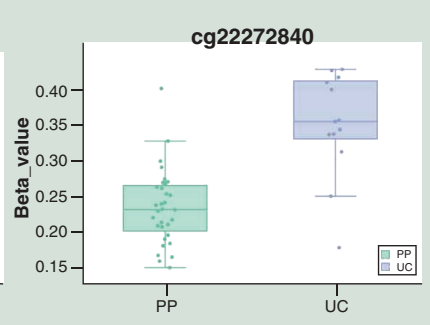

cg23394673
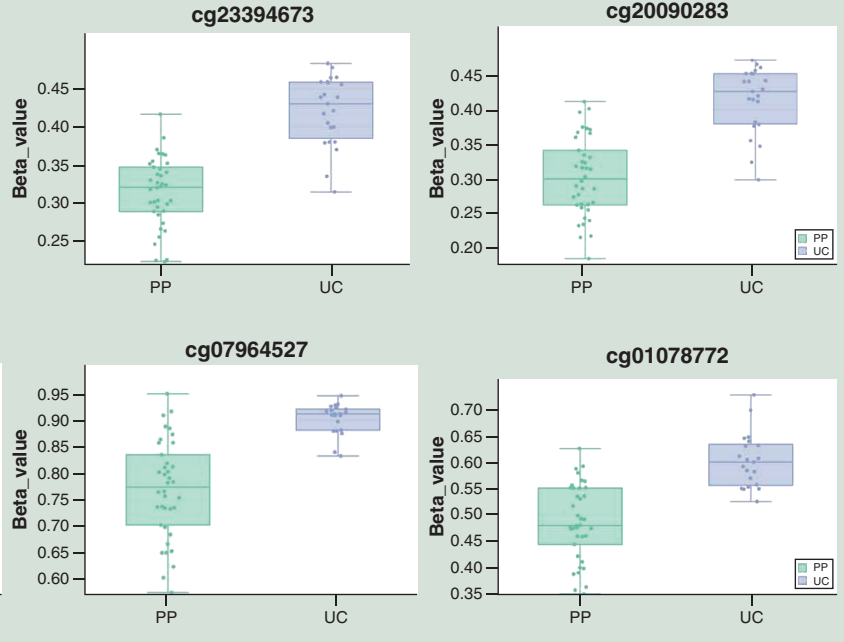

Figure 2. DNA methylation level of the most significant $\mathrm{CpG}$ site in psoriatic skin versus normal skin samples corresponding locations. (A-C) PP is highlighted in green, NN are depicted in blue. $\beta$-value: DNA methylation levels.

Table 1. Ten location-specific differential methylation sites were validated by bisulfate conversion sequencing.

\begin{tabular}{|lllllllll|}
\hline Body location & $\begin{array}{l}\text { CpG loci } \\
\text { annotation }\end{array}$ & Target & Chr & $\begin{array}{l}\text { Genome } \\
\text { position }\end{array}$ & Distance to TSS & Average PP & Average NN & p-value \\
\hline Back & cg22272840 & SOX7 & 8 & $10,586,882$ & 1202 & 0.22 & 0.34 & $3.75 \mathrm{E}-06$ \\
\hline & cg11910652 & TJAP1 & 6 & $43,457,275$ & -46 & 0.80 & 0.71 & $1.21 \mathrm{E}-03$ \\
\hline & cg04883026 & SPTBN2 & 11 & $66,487,938$ & 932 & 0.35 & 0.46 & $4.77 \mathrm{E}-05$ \\
\hline Extremities & cg23394673 & TNFRSF4 & 1 & $1,149,212$ & 336 & 0.27 & 0.35 & $4.97 \mathrm{E}-06$ \\
\hline & cg20090283 & SRCIN1 & 17 & $36,718,550$ & 43,633 & 0.15 & 0.19 & $2.00 \mathrm{E}-02$ \\
\hline & cg07852840 & SPSB4 & 3 & $140,813,919$ & 43,676 & 0.49 & 0.30 & $3.65 \mathrm{E}-06$ \\
\hline & cg02479022 & SLC39A14 & 8 & $22,250,005$ & -337 & 0.50 & 0.32 & $4.55 \mathrm{E}-06$ \\
\hline Abdomen & cg07964527 & PDE8A & 15 & $85,534,864$ & 9660 & 0.69 & 0.83 & $5.95 \mathrm{E}-05$ \\
\hline & cg22729681 & PBX1 & 1 & $164,717,625$ & 189,029 & 0.49 & 0.35 & $2.06 \mathrm{E}-05$ \\
\hline & cg01866518 & PXN & 12 & $120,673,236$ & 14,728 & 0.64 & 0.50 & $1.42 \mathrm{E}-06$ \\
\hline
\end{tabular}

Chr: Chromosome number; NN: Normal skin; PP: Psoriatic skin; TSS: Transcription start site.

A total of 247 common-shared sites were identified among the three locations, 58.2\% (144) of which overlapped with DMSs identified in previous publications [3]. The top 25 sites are listed in Table 2. The 247 DMSs uniquely mapped to 178 genes, 146 of which were located in the gene body (Figure $1 \&$ Supplementary Table 7). In addition, KEGG pathway analysis showed that the 178 genes are significantly enriched in 'regulation of actin cytoskeleton' and 'proteoglycans in cancer' (Supplementary Table 8). 


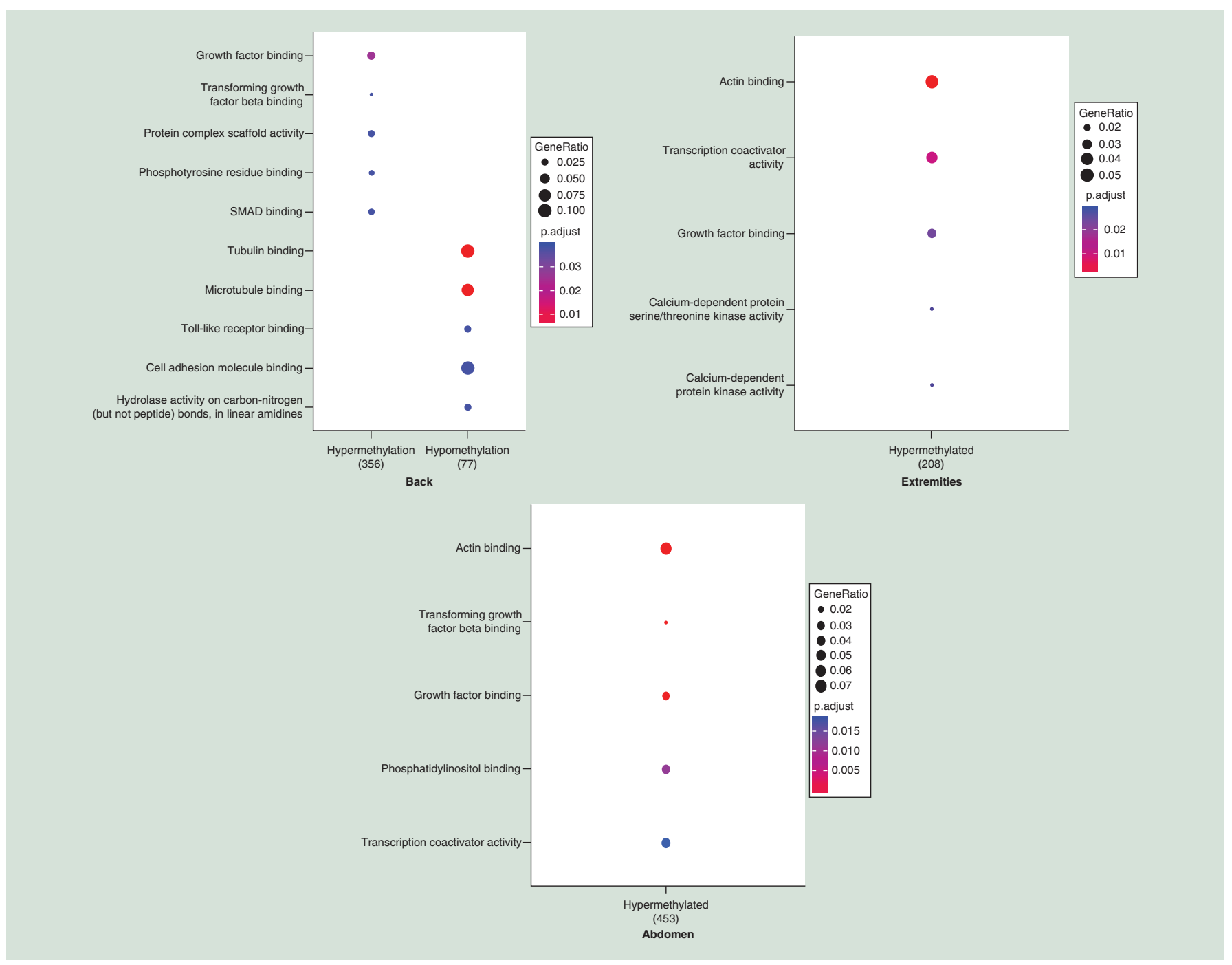

Figure 3. Gene Ontology comparative analysis with differentially methylated genes across the three locations. Each circle is colored in accordance with p-value. Size of a circle as a proportional adjustment to geneRatio size. The abscissa of each circle corresponds to the differential methylation gene, and the ordinate corresponds to the relevant gene functional region.

\section{Histopathological features related to location-specific DMSs}

We performed pathological examination of all the $108 \mathrm{PP}$ samples, and collected data pertaining to physiological features, including hyperkeratosis, Munro's microabscess, parakeratosis, elongation of rete pegs, focal hypergranulosis, thinning of granulosa, thickening of acanthosis and vascular dilatation and congestion. We compared PP samples with and without histopathological features and found that the extremities are more prone to hyperkeratosis than the back and abdomen (Table 3). No significant DMSs were identified between hyperkeratosis-present and -absent groups. We further investigated whether the histopathological features affected location-specific DMSs among the three body locations, and observed hyperkeratosis was closely related to some extremity-specific DMSs, suggesting a contribution to body location differences in psoriasis. For example, the methylation level of cg21942490 in extremities is significantly lower than in the back or abdomen. This locus is located in 1500 bp upstream of the HOXA9 transcription start site, possibly indicating variable roles of HOXA9 across different anatomical body locations. We also found some loci of the $H O X$ family genes displaying methylation changes in the $\mathrm{PP}_{\text {extremity }}$ versus $\mathrm{PP}_{\text {back }}$ and $\mathrm{PP}_{\text {extremity }}$ versus $\mathrm{PP}_{\text {abdomen }}$ comparisons, further suggesting the contribution of the $H O X$ family to hyperkeratosis. By integrating our previous RNA-seq dataset of 20 samples: 4 extremities, 11 back and 5 abdomen, we found that HOXB9 and HOXD4 were more downregulated in the extremity than in the abdomen or back, even in such a small sample (Table 4). However, we did not find significant differences from the paired analysis of PASI scores among 
Table 2. Top 25 unique mapped genes identified among back, extremities and abdomen.

\begin{tabular}{|c|c|c|c|c|c|c|c|c|c|}
\hline \multirow[t]{2}{*}{ DMS } & \multirow[t]{2}{*}{ Chr } & \multirow[t]{2}{*}{ Gene $^{\dagger}$} & \multirow[t]{2}{*}{ Context $^{\ddagger}$} & \multicolumn{2}{|c|}{ Back } & \multicolumn{2}{|c|}{ Extremities } & \multicolumn{2}{|c|}{ Abdomen } \\
\hline & & & & p-value ${ }^{\S}$ & $\beta^{\mathbb{I}}$ & p-value ${ }^{\S}$ & $\beta^{\mathbb{I}}$ & p-value ${ }^{\S}$ & $\boldsymbol{\beta}^{\mathbb{I}}$ \\
\hline cg22724943 & 1 & MAN1C1 & Body & $8.31 \mathrm{E}-06$ & -0.137 & $2.85 \mathrm{E}-08$ & -0.170 & $1.28 \mathrm{E}-04$ & -0.153 \\
\hline cg04930596 & 12 & NCOR2 & Body & 4.46E-04 & -0.102 & $3.86 \mathrm{E}-08$ & -0.155 & $5.22 \mathrm{E}-04$ & -0.137 \\
\hline cg01678309 & 15 & CORO2B & Body & $2.26 \mathrm{E}-05$ & -0.114 & $3.89 \mathrm{E}-08$ & -0.130 & $2.50 \mathrm{E}-04$ & -0.119 \\
\hline cg13855435 & 17 & YPEL2 & Body & $1.97 \mathrm{E}-04$ & -0.119 & $3.89 \mathrm{E}-08$ & -0.150 & $6.83 \mathrm{E}-04$ & -0.131 \\
\hline cg09490124 & 7 & PUS7 & Body & $2.00 \mathrm{E}-04$ & -0.110 & $6.42 \mathrm{E}-08$ & -0.143 & 2.07E-04 & -0.145 \\
\hline cg12636499 & 10 & $S H 3 P X D 2 A$ & Body & $4.60 \mathrm{E}-05$ & -0.118 & $7.73 \mathrm{E}-08$ & -0.152 & $1.16 \mathrm{E}-04$ & -0.160 \\
\hline cg14541011 & 9 & RALGDS & Body & $1.06 \mathrm{E}-04$ & -0.143 & $7.73 \mathrm{E}-08$ & -0.185 & $6.25 \mathrm{E}-04$ & -0.155 \\
\hline $\operatorname{cg} 10926487$ & 17 & TIMP2 & Body & 4.65E-04 & -0.102 & $8.09 \mathrm{E}-08$ & -0.148 & $3.19 \mathrm{E}-04$ & -0.134 \\
\hline cg13086983 & 1 & ECE1 & Body & $1.18 \mathrm{E}-05$ & -0.142 & $8.61 \mathrm{E}-08$ & -0.156 & $1.43 \mathrm{E}-04$ & -0.156 \\
\hline cg18735015 & 10 & $S H 3 P X D 2 A$ & Body & $3.08 \mathrm{E}-04$ & -0.112 & $8.61 \mathrm{E}-08$ & -0.172 & $3.75 \mathrm{E}-04$ & -0.146 \\
\hline cg22330763 & 6 & SLC29A1 & $5^{\prime}$ UTR & $2.94 \mathrm{E}-04$ & -0.111 & $8.61 E-08$ & -0.162 & 2.77E-04 & -0.144 \\
\hline cg01912455 & 22 & SMARCB1 & TSS1500 & $3.95 \mathrm{E}-05$ & -0.126 & $9.54 \mathrm{E}-08$ & -0.146 & $1.77 \mathrm{E}-04$ & -0.159 \\
\hline cg02442900 & 2 & $D G K D$ & Body & $9.48 \mathrm{E}-06$ & -0.114 & $9.54 \mathrm{E}-08$ & -0.125 & $1.50 \mathrm{E}-04$ & -0.127 \\
\hline cg03052794 & 19 & C19orf22 & Body & $1.51 \mathrm{E}-05$ & 0.110 & $9.54 \mathrm{E}-08$ & 0.120 & $1.87 \mathrm{E}-04$ & 0.109 \\
\hline cg17825194 & 12 & NCOR2 & Body & $1.15 \mathrm{E}-04$ & -0.101 & $9.54 \mathrm{E}-08$ & -0.139 & $3.99 \mathrm{E}-04$ & -0.130 \\
\hline $\operatorname{cg} 23601468$ & 22 & SYN3 & Body & $1.75 \mathrm{E}-05$ & -0.133 & $9.54 \mathrm{E}-08$ & -0.140 & $1.49 \mathrm{E}-04$ & -0.146 \\
\hline $\operatorname{cg} 24216770$ & 20 & DLGAP4 & Body & $5.60 \mathrm{E}-05$ & -0.133 & $9.54 \mathrm{E}-08$ & -0.146 & $2.78 \mathrm{E}-04$ & -0.143 \\
\hline cg10863207 & 17 & RFFL & $5^{\prime}$ UTR & $2.38 \mathrm{E}-05$ & 0.161 & $1.00 \mathrm{E}-07$ & 0.199 & $3.08 \mathrm{E}-04$ & 0.161 \\
\hline cg02550308 & 16 & SNTB2 & Body & $1.36 \mathrm{E}-04$ & -0.117 & $1.09 \mathrm{E}-07$ & -0.135 & $5.66 \mathrm{E}-04$ & -0.119 \\
\hline cg04099543 & 11 & P2RYG & $5^{\prime}$ UTR & $9.10 \mathrm{E}-05$ & 0.122 & 1.19E-07 & 0.155 & 3.33E-04 & 0.136 \\
\hline cg11829253 & 15 & PSTPIP1 & Body & $1.57 \mathrm{E}-04$ & -0.110 & $1.26 \mathrm{E}-07$ & -0.123 & 4.14E-04 & -0.110 \\
\hline cg12196406 & 19 & FXYD1 & TSS200 & 4.53E-04 & -0.101 & $1.26 \mathrm{E}-07$ & -0.127 & 1.10E-04 & -0.136 \\
\hline cg11826961 & 17 & THRA & $5^{\prime}$ UTR & $1.52 \mathrm{E}-04$ & -0.116 & $1.27 \mathrm{E}-07$ & -0.145 & $3.16 \mathrm{E}-04$ & -0.147 \\
\hline cg05049448 & 7 & SNX8 & Body & $3.67 \mathrm{E}-04$ & -0.115 & $1.32 \mathrm{E}-07$ & -0.153 & $4.83 \mathrm{E}-04$ & -0.135 \\
\hline cg00288598 & 8 & EIF2C2 & Body & 4.63E-05 & 0.182 & $1.34 \mathrm{E}-07$ & 0.213 & 2.69E-04 & 0.173 \\
\hline \multicolumn{10}{|c|}{$\begin{array}{l}\text { † Gene: gene name, according to the HUGO Gene Nomenclature Committee. } \\
\text { ‡Context: location of the gene-associated CpG-site(s) with respect to the gene context. } \\
\text { §Adjusted p-value: t-test p-values adjusted for Benjamini-Hochberg corrections. } \\
\mathbb{I}_{\delta} \text { : methylation differences between the cases and controls. } \\
\text { Chr: Chromosome number; DMS: Differentially methylated site. }\end{array}$} \\
\hline
\end{tabular}

\begin{tabular}{|c|c|c|c|c|c|c|c|c|c|}
\hline \multirow[t]{2}{*}{ Physiology features } & \multicolumn{2}{|c|}{ Back } & \multicolumn{2}{|c|}{ Extremities } & \multicolumn{2}{|c|}{ Abdomen } & \multicolumn{3}{|c|}{ p-values: statistic significance } \\
\hline & Present & Absent & Present & Absent & Present & Absent & $\begin{array}{l}\text { Back vs } \\
\text { extremities }\end{array}$ & $\begin{array}{l}\text { Back vs } \\
\text { abdomen }\end{array}$ & $\begin{array}{l}\text { Extremities vs } \\
\text { abdomen }\end{array}$ \\
\hline Hyperkeratosis & 25 & 7 & 36 & 2 & 29 & 9 & 0.038 & 0.85 & 0.022 \\
\hline Parakeratosis & 23 & 9 & 35 & 3 & 31 & 7 & 0.025 & 0.33 & 0.17 \\
\hline Munro microabscess & 12 & 20 & 9 & 29 & 8 & 30 & 0.21 & 0.13 & 0.78 \\
\hline Elongation of rete pegs & 14 & 18 & 11 & 27 & 16 & 22 & 0.19 & 0.89 & 0.23 \\
\hline Focal hypergranulosis & 0 & 32 & 1 & 37 & 1 & 37 & $\mathrm{~N} / \mathrm{A}$ & $\mathrm{N} / \mathrm{A}$ & $\mathrm{N} / \mathrm{A}$ \\
\hline Granulosa thinning & 5 & 27 & 10 & 28 & 10 & 28 & 0.28 & 0.28 & 1 \\
\hline Acanthosis & 23 & 9 & 31 & 7 & 25 & 13 & 0.33 & 0.58 & 0.11 \\
\hline $\begin{array}{l}\text { Vascular dilatation and } \\
\text { congestion }\end{array}$ & 25 & 7 & 34 & 4 & 33 & 5 & 0.19 & 0.33 & 0.72 \\
\hline
\end{tabular}

different body locations. 


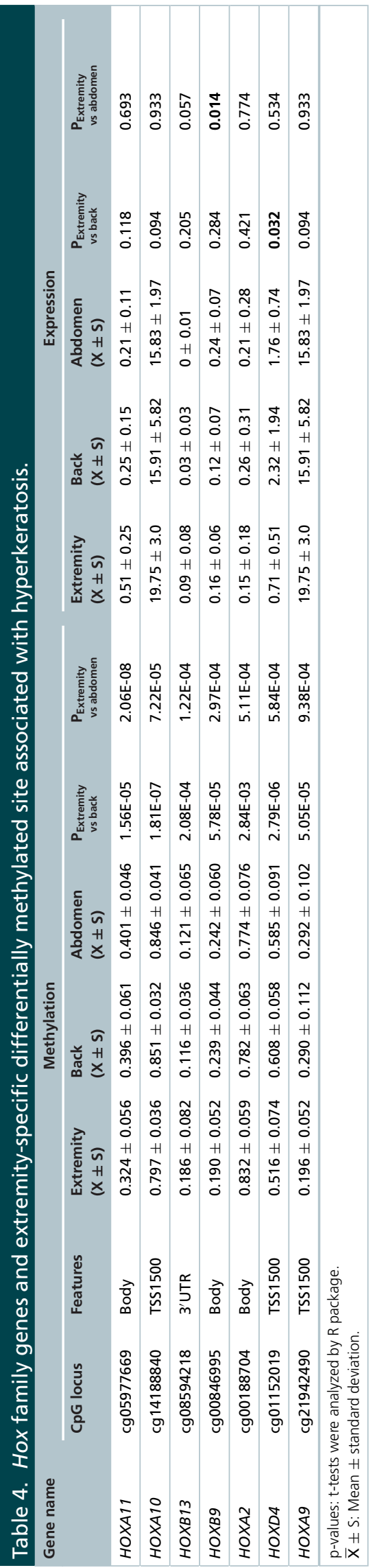




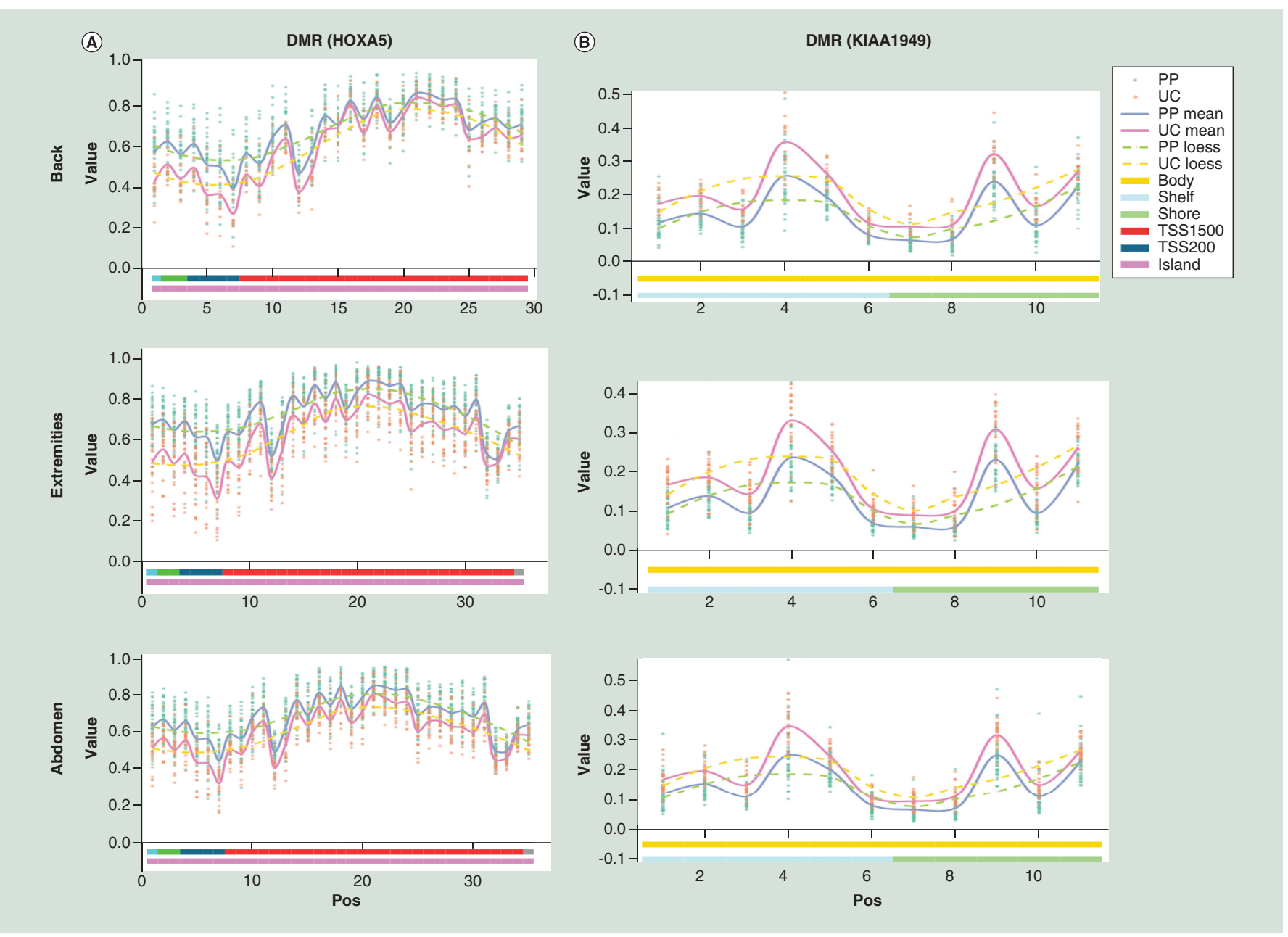

Figure 4. DNAm level distribution of the CpG sites for two common genes identified in the two most significant shared differentially methylated regions (DMR1 and DMR2) across the three locations. The $y$-axis denotes the DNA methylation level and the $x$-axis is the CpG sites in HOXA5, and the green dot represents PP, NN are depicted in red. Blue and pink solid wavy lines represent PP mean and UC mean, respectively. PP loess and UC loess are depicted as brownish green and yellow, respectively. These CpG loci correspond to the gene region below the abscissa, whereas the color of each gene region is consistent with the corresponding annotation in the upper right corner. (Light blue: 1st Exon; light green: 5'UTR; Red: TSS1500; dark blue: TSS200; pink: island). The description of KIAA1949 gene is almost identical to the characterization of the above HOXA5 gene.

DMR: Differentially methylated region; PP: Psoriatic skin.

Differences in DMRs between extremities, back \& abdomen in psoriasis

Finally, to explore whether the region of methylation affected psoriasis, we used the Bumphunter algorithm to detect DMRs in different locations. A total of 457 DMRs were identified, of which 108 (23.6\%) mapped to the back areas, whereas the remaining 198 (43.2\%) and 151 (33.0\%) mapped to the abdomen and extremities, respectively. There were 34 common DMRs present at all three locations (Supplementary Table 9).

Here we showed the two most representative DMRs $\left(\mathrm{p}<1.86 \times 10^{-5}\right)$ that overlap HOXA5 and KIAA1949. Specifically, the HOXA5 DMRs are hypermethylated, whereas the KIAA1949 DMRs are hypomethylated, in PP compared with NN (Figure 4). The starting and ending point of HOXA5 DMRs ranged from 27,183,196 to 2,718,4821 on chromosome 7, which contained 35 CG sites; while the starting and ending point of KIAA1949 DMRs ranged from 30,652,202 to 30,652,829 on chromosome 6, which included 11 CG sites. Both these DMRs are related to gene function and development. After annotating the shared HOXA5 DMRs harboring DMSs, we found that about 70\% of CpGs were located on CpG islands and TSS1500, whereas the CpGs of KIAA1949 DMRs were located in non-CGI regions and gene body (Figure 4). Furthermore, our result indicate that the expression of HOXA5 $\left(\mathrm{p}=6.55 \times 10^{-2}\right)$ and KIAA1949 was not significant. 


\section{Discussion}

Our previous genome-wide methylation study identified strong associations between nine CpG loci and psoriasis [3]. Here for the first time, we separately analyzed the genome-wide DNAm profiles of skin samples from the three anatomical body locations, that is, back, extremities and abdomen. We focused on the disparate locations of the psoriatic plaques, because psoriatic lesions in disparate locations of psoriasis show different severity and differ in response to treatment, a phenomenon that may be associated with abnormal DNA methylation [8,14]. Because DNAm variation affects the expression of genes or nearby genes with positive or negative correlations, DNAm has become a research hotspot in the pathogenesis of psoriasis [3,24].

We observed extensive differences in DNAm in PP skin compared with NN across three somatic locations and also found that 178 shared unique mapped sites were strongly associated with psoriasis. However, these sites mainly occurred within the gene body and not in the promoters, indicating that these loci might be involved in the regulation of gene expression and related to tissue or cell development discrepancies previously identified [11,12,25,26]. The differences in DNAm are highly specific among distinct locations, further molding specific function pattern of these differentiated tissues. About $42 \%$ of the common-shared sites we identified differ from the original data because of our small sample sizes and methodological discrepancies as well as the incorporation of data from heterogeneous locations.

We performed GO analysis on differentially methylated genes in each location and found the same hypermethylated gene functional enrichment region annotated with 'growth factor binding' as well as specific regions. Growth factors closely related to excessive hyperproliferation and differentiation of psoriatic keratinocytes and blood vessels, such as VEGF [27], IGF I [28], FGF [29,30], NGF-regulating neuropeptides and angiogenesis [31], were encoded by hypermethylated genes and almost exclusively secreted by inflammatory T-cell-dominated psoriatic pathogenesis [32]. The results were similar to those previously reported by Park et al. [33], indicating that the hypermethylation pattern of $\mathrm{CD}^{+} \mathrm{T}$ cells in psoriatic lesions caused epidermal and vascular proliferation of psoriatic lesions. It appears that these enriched hypermethylated genes may be involved in the regulation of growth factor expression in psoriasis. Meanwhile, we also identified specific processes among them. Differences in GO analysis results at each location may be related to differences in gene expression, further triggering a variation in inflammation. Furthermore, we found specific hypomethylated gene enrichment in the back, but not in the limbs and abdomen, suggesting that these specifically enriched genes can be considered as location-specific markers. Consistent with previous findings, hypomethylated genes are more likely to be linked to tissue-specific functions [12]. From the results of our study, it appears that shared biological pathways connected with development were enriched in different locations of psoriasis.

The location-specific methylation genes-SOX7 in the back, TNFRSF4 in the extremities and PDE8A in the abdomen - could be used as specific markers for functional annotations, such as enhancers, DNase I hypersensitivity sites and transcription factors, and used in comparison studies to further understand the pathogenesis of psoriasis in different body locations. Previous study has shown that the $S O X 7$ is expressed specifically in endothelial cells of vessel, providing positive feedback to VEGF signaling via the mTOR pathway [23]. TNFRSF4, known as OX4O in $\mathrm{CD} 4^{+} \mathrm{T}$ cells, is motivated by the NF- $\kappa \mathrm{B}$ signaling pathway to increase the expression of antiapoptotic molecules, whereas NF- $\mathrm{B}$ signaling pathway is closely associated with epidermal proliferation and differentiation $[20,34]$. The abdomen-specific PDE8A acted as a potential drug target gene regulating $\mathrm{CD}^{+} \mathrm{T}$ cell function via the $P D E 8 A-R a f-1$ kinase signaling pathway [35]. These results show that efforts to develop and test inhibitors of these location-specific gene pathways could provide a novel and efficient way to treat psoriasis.

The DNAm profile varies between different anatomical locations, possibly because the infiltration of inflammatory cells in psoriatic lesions and different pathological features change tissue components and DNAm [12,36]. Previous study has shown that DNAm and pivotal pathogenic genes might be involved in regulating the cardinal histopathological features detected in psoriasis [15]. Although significant DMSs were not observed between PP and NN at the same locations with or without pathological characteristics, we found extremity-specific DMSs associated with hyperkeratosis among the three locations. Both are caused due to comparative analysis by choosing $\mathrm{PP}$ versus $\mathrm{NN}$ or other confounding factors, such as cellular heterogeneity, interindividual discrepancy and the limited presence of conspicuous pathological characteristics. Furthermore, the detection of some HOX family gene loci related to hyperkeratosis as well as the extremity-specific differential methylation site cg21942490 located on HOXA9 further indicate that $H O X$ family gene DMSs mediated unique psoriatic pathological characteristics at different locations. Our gene expression analyses showed that HOXB9 and HOXD4 were downregulated in the 
extremities. These data indicate that the expression of the HOX9 paralogues HOXA9 and HOXB9 is related to FGFs in a limb-specific manner [37-39]. We observed no significant differences among PASI scores in these locations, which could be related to a decreased severity of psoriatic lesions. However, a recent study found that $\mathrm{T}$ lymphocyte infiltration can affect psoriatic epidermal differentiation and proliferation, and change the composition of each layer [36. Some defects in the PASI score and histopathology of our original samples made it infeasible to further explore the correlation between DNAm and lymphocyte infiltration level from different locations. In future, we intend to pursue further research using appropriate samples.

Furthermore, we identified the most common HOXA5 DMRs and KIAA1949DMRs. HOXA5 is a member of the $H O X$ gene family, which encodes a transcription factor controlling development and differentiation, which specifies regional differences along the anterior-posterior axis [40,41]. We found that HOXA5 is a hypermethylated gene in PP versus $\mathrm{NN}$, as found in previous study [42]. However, the HOXA5 expression that we observed was inconsistent with that reported by Li B team [43], probably demonstrating the limitations imposed by our small sample size. Moreover, HOXA5 is also underexpressed in some human tumors, including gastric cancer [44], colorectal cancer [45], breast cancer [46], liposarcomas [47] and, most notably, chronic myeloid leukemia [48], in which HOXA5 hypermethylation was the target of gene inactivation. In addition, the presence of HOXA5-induced apoptosis indicates that the HOXA5 gene may have the ability to activate antagonist genes against the release of growth factors, which mainly implicates inhibition of epidermal formation and keratinocyte differentiation [49].

As a novel candidate hypomethylated gene implicated in psoriasis, the KIAA1949 encoded phosphatase $1 \mathrm{~F}$-actin cytoskeleton targeting subunit protein. Previous studies have shown that KIAA1949, which is located between the $H L A-C$ and $H L A-E$ gene clusters on human chromosome 6, associates with protein phosphatase 1 capable of modulating actin dynamics [50,51]. The role of KIAA1949 in the pathogenesis of psoriasis has not yet been reported. Therefore, the KIAA1949 gene expression in our study is not significant, but its expression is low in breast cancer [52]. Our results indicate that the differences in DMRs at different body locations are involved in the pathogenesis of psoriasis.

\section{Conclusion}

We have shown that location-specific and location-shared DMSs and DMRs contribute to our understanding of psoriatic pathogenesis. Although the DNAm and pathological data that we collected had some limitations, our results, validated using external datasets, suggest the presence of a number of location-specific DMSs that are associated with psoriatic histopathological features. In particular, the HOX family genes are closely correlated with hyperkeratosis, with HOXA9 being specific to the extremities. DMRs analysis among the three locations identified the HOXA5 and KIAA1949 regions as potentially vital to the modulation of keratinocyte development in psoriasis. We were also able to show that growth factors contribute to psoriatic pathological changes. Identification of the common and location-special genes involved in regulation of psoriatic pathogenesis may be valuable in developing drugs for the better treatment of psoriasis. Our results need to be further developed using a larger sample size and additional pathological HOX family characteristics.

\section{Future perspective}

The data analysis and functional studies of DMSs and DMRs are particularly important in the investigation of psoriasis in different locations. By analyzing the histopathology of DMSs and DMRs in psoriasis in different locations, we identified the similarities and differences among the different locations. These similarities and differences will help in deepening our understanding of the pathogenesis of psoriasis and could provide a basis for the development of new drugs. Further research should focus on the mechanism by which the HOX family gene plays a regulatory role in psoriatic pathogenesis in different locations. This can be achieved by increasing the sample size and using more appropriate pathological tissues, for example, in PP versus PN. It should then be possible to select multiple overlapped $H O X$ genes with differential expression among the three locations and perform pathway and functional analysis. These location-specific and location-shared differential methylation gene function and pathways could potentially contribute to the development of tailored responses to highly targeted agents. 
processed the data and performed the statistical analyses. M Wu and F Zhou drafted the manuscript. All authors reviewed the results and contributed to the final manuscript.

\section{Acknowledgments}

The authors are grateful to all the participants in this study.

Financial \& competing interests disclosure

This study was mainly supported by the National Natural Science Foundation of China (31671307), the Scientific Research of BSKY of Anhui Medical University (XJ201634) and the Key Program of Outstanding Talents support of Anhui province (gxyqZD2017028).

The authors have no other relevant affiliations or financial involvement with any organization or entity with a financial interest in or financial conflict with the subject matter or materials discussed in the manuscript apart from those disclosed.

No writing assistance was utilized in the production of this manuscript.

Ethical conduct of research

Written informed consent was obtained from all of the subjects, under an Anhui Medical University approved protocol, and the study was performed according to the Declaration of Helsinki principles.

\section{Open access}

This work is licensed under the Attribution-NonCommercial-NoDerivatives 4.0 Unported License. To view a copy of this license, visit http://creativecommons.org/licenses/by-nc-nd/4.0/

\section{Availability of data \& materials}

The epigenome-wide data generated and analyzed during the current study are available from the corresponding author on reasonable request. The raw data had been submitted to Gene Expression Omnibus under accession GSE73894.

\section{Summary points}

- We revealed 315, 291 and 801 location-specific differentially methylated sites (DMS) for back, extremities and abdomen, respectively.

- We confirmed the reliability of these location-specific DMSs by bisulfite sequencing, such as SOX7 in the back, TNFRSF4 in the extremities and PDE8A in the abdomen.

- These location-specific methylated genes were significantly enriched in 'cell adhesion molecule binding', 'transcription coactivator activity' and 'phosphatidylinositol binding'.

- We also found 178 location-common differentially methylated genes and referred genes were enriched in 'growth factor binding' as implicated by Gene Ontology analysis.

- Kyoto Encyclopedia of Genes and Genomes (KEGG) pathway analysis showed that 178 unique-mapped genes were significantly enriched in 'regulation of actin cytoskeleton' and 'proteoglycans in cancer'.

- We observed that hyperkeratosis is tightly related to some extremity-specific DMSs, especially for the extremity-specific differential methylation site cg21942490 located on HOXA9, possibly indicating variable roles of HOXA9 across different anatomical body regions.

- We further observed 457 differential methylation regions, in which 23.6, 43.2 and 33.0\% were mapped to back, abdomen and extremities, respectively. HOXA5 and KIAA1949 are the top overlapped differential methylation regions.

- $H O X B 9$ and HOXD4 were more downregulated in the extremity than in the abdomen or back, but the expression of HOXA5 and KIAA1949 gene was not significant.

\section{References}

Papers of special note have been highlighted as: $\bullet$ of interest; $\bullet \bullet$ of considerable interest

1. Perera GK, Di Meglio P, Nestle FO. Psoriasis. Annu. Rev. Pathol. 7, 385-422 (2012).

2. Parisi R, Symmons DP, Griffiths CE, Ashcroft DM. Global epidemiology of psoriasis: a systematic review of incidence and prevalence. J. Invest. Dermatol. 133(2), 377-385 (2013).

3. Zhou F, Wang W, Shen C et al. Epigenome-wide association analysis identified nine skin DNA methylation loci for psoriasis. J. Invest. Dermatol. 136(4), 779-787 (2016).

- Allows to understand the differences in DNA methylation (DNAm) levels and their expression associations in patients with psoriasis from the perspective of genome-wide level. 
4. Roberson ED, Liu Y, Ryan C et al. A subset of methylated CpG sites differentiate psoriatic from normal skin. J. Invest. Dermatol. 132(3 Pt 1), 583-592 (2012).

5. Zhang P, Zhao M, Liang G et al. Whole-genome DNA methylation in skin lesions from patients with psoriasis vulgaris. J. Autoimmun. 41, 17-24 (2013).

- Allows to understand differentially methylated regions and their function in patients with psoriasis from the perspective of genome-wide DNAm patterns.

6. Chen K, Wang G, Jin $\mathrm{H}$ et al. Clinic characteristics of psoriasis in china: a nationwide survey in over 12000 patients. Oncotarget 8(28), 46381-46389 (2017).

7. Hagg D, Sundstrom A, Eriksson M, Schmitt-Egenolf M. Severity of psoriasis differs between men and women: a study of the clinical outcome measure psoriasis area and severity index (PASI) in 5438 Swedish register patients. Am. J. Clin. Dermatol. 18(4), 583-590 (2017).

8. Gu X, Nylander E, Coates PJ, Fahraeus R, Nylander K. Correlation between reversal of DNA methylation and clinical symptoms in psoriatic epidermis following narrow-band UVB phototherapy. J. Invest. Dermatol. 135(8), 2077-2083 (2015).

-• Allows to learn that DNAm pattern was reversed at the end of narrow-band UVB phototherapy in psoriatic epidermis showing excellent clinical improvement.

9. Ai R, Hammaker D, Boyle DL et al. Joint-specific DNA methylation and transcriptome signatures in rheumatoid arthritis identify distinct pathogenic processes. Nat. Commun. 7, 11849 (2016).

-. The report shows differences in biological pathways and DNA signatures between rheumatoid arthritis knee and hip fibroblast-like synoviocytes.

10. Schultz MD, He Y, Whitaker JW et al. Human body epigenome maps reveal noncanonical DNA methylation variation. Nature 523(7559), 212-216 (2015).

11. Deaton AM, Webb S, Kerr ARW et al. Cell type-specific DNA methylation at intragenic CpG islands in the immune system. Genome Res. 21(7), 1074-1086 (2011).

12. Lokk K, Modhukur V, Rajashekar B et al. DNA methylome profiling of human tissues identifies global and tissue-specific methylation patterns. Genome Biol. 15(4), r54 (2014).

13. Zhang B, Zhou Y, Lin N et al. Functional DNA methylation differences between tissues, cell types, and across individuals discovered using the M\&M algorithm. Genome Res. 23(9), 1522-1540 (2013).

-. Allows to learn that there were no differences in gene expression profile among the locations of psoriatic plaques. While the gene expression profiles of involved psoriatic skin correlated with psoriasis area and severity index score.

14. Quekenborn-Trinquet V, Fogel P, Aldana-Jammayrac $\mathrm{O}$ et al. Gene expression profiles in psoriasis: analysis of impact of body site location and clinical severity. Br. J. Dermatol. 152(3), 489-504 (2005).

-. Makes realize that there were differences in the DNAm distribution and function of different tissues or cells in human body.

15. Chandra A, Senapati S, Roy S, Chatterjee G, Chatterjee R. Epigenome-wide DNA methylation regulates cardinal pathological features of psoriasis. Clin. Epigenetics 10(1), 108 (2018).

16. Teschendorff AE, Marabita F, Lechner $\mathrm{M}$ et al. A $\beta$-mixture quantile normalization method for correcting probe design bias in Illumina Infinium 450 k DNA methylation data. Bioinformatics 29(2), 189-196 (2013).

17. Morris TJ, Butcher LM, Feber A et al. Champ: $450 \mathrm{k}$ chip analysis methylation pipeline. Bioinformatics 30(3), 428-430 (2014).

18. Gentleman RC, Carey VJ, Bates DM et al. Bioconductor: open software development for computational biology and bioinformatics. Genome Biol. 5(10), R80 (2004).

19. Croft M. The role of tnf superfamily members in T-cell function and diseases. Nat. Rev. Immunol. 9(4), 271-285 (2009).

20. Kaufman CK, Fuchs E. It's got you covered. NF-кB in the epidermis. J. Cell Biol. 149(5), 999-1004 (2000).

21. Dong H, Osmanova V, Epstein PM, Brocke S. Phosphodiesterase 8 (PDE8) regulates chemotaxis of activated lymphocytes. Biochem. Biophys. Res. Commun. 345(2), 713-719 (2006).

22. Vang AG, Ben-Sasson SZ, Dong H et al. PDE8 regulates rapid TEFF cell adhesion and proliferation independent of ICER. PLoS ONE 5(8), e12011 (2010).

23. Kim K, Kim IK, Yang JM et al. Soxf transcription factors are positive feedback regulators of VEGF signaling. Circ. Res. 119(7), 839-852 (2016).

24. Izzi B, Pistoni M, Cludts K et al. Allele-specific DNA methylation reinforces PEAR1 enhancer activity. Blood 128(7), 1003-1012 (2016).

25. Maunakea AK, Nagarajan RP, Bilenky M et al. Conserved role of intragenic DNA methylation in regulating alternative promoters. Nature 466(7303), 253-257 (2010).

26. Baribault C, Ehrlich KC, Ponnaluri VKC et al. Developmentally linked human DNA hypermethylation is associated with down-modulation, repression, and upregulation of transcription. Epigenetics 13(3), 275-289 (2018).

27. Vegfors J, Ekman AK, Stoll SW, Bivik Eding C, Enerback C. Psoriasin (S100A7) promotes stress-induced angiogenesis. Br. J. Dermatol. 175(6), 1263-1273 (2016). 
28. Wraight CJ, White PJ, Mckean SC et al. Reversal of epidermal hyperproliferation in psoriasis by insulin-like growth factor I receptor antisense oligonucleotides. Nat. Biotechnol. 18(5), 521-526 (2000).

29. Kovacs D, Falchi M, Cardinali G et al. Immunohistochemical analysis of keratinocyte growth factor and fibroblast growth factor 10 expression in psoriasis. Exp. Dermatol. 14(2), 130-137 (2005).

30. Cheng $\mathrm{H}, \mathrm{Xu}$ M, Liu X et al. TWEAK/Fn14 activation induces keratinocyte proliferation under psoriatic inflammation. Exp. Dermatol. 25(1), 32-37 (2016).

31. Raychaudhuri SK, Raychaudhuri SP. NGF and its receptor system: a new dimension in the pathogenesis of psoriasis and psoriatic arthritis. Ann. N Y Acad. Sci. 1173, 470-477 (2009).

32. Deng Y, Chang C, Lu Q. The inflammatory response in psoriasis: a comprehensive review. Clin. Rev. Allergy Immunol. 50(3), 377-389 (2016).

33. Park GT, Han J, Park SG, Kim S, Kim TY. DNA methylation analysis of CD4 ${ }^{+}$T cells in patients with psoriasis. Arch. Dermatol. Res. 306(3), 259-268 (2014).

34. Rogers PR, Song J, Gramaglia I, Killeen N, Croft M. Ox40 promotes BCL-XL and BCL-2 expression and is essential for long-term survival of CD4 T cells. Immunity 15(3), 445-455 (2001).

35. Basole CP, Nguyen RK, Lamothe K et al. PDE8 controls CD4 $\left(^{+}\right) \mathrm{T}$ cell motility through the PDE8A-RAF-1 kinase signaling complex. Cell Signal. 40, 62-72 (2017).

36. Lorthois I, Simard M, Morin S, Pouliot R. Infiltration of T cells into a three-dimensional psoriatic skin model mimics pathological key features. Int. J. Mol. Sci. (2019). https://www.ncbi.nlm.nih.gov/pmc/articles/PMC6479293/

37. Cohn MJ, Patel K, Krumlauf R et al. HOX9 genes and vertebrate limb specification. Nature 387(6628), 97-101 (1997).

38. Xu B, Wellik DM. Axial HOX9 activity establishes the posterior field in the developing forelimb. Proc. Natl Acad. Sci. USA 108(12), 4888-4891 (2011).

39. Raines AM, Magella B, Adam M, Potter SS. Key pathways regulated by HOXA9,10,11/HOXD9,10,11 during limb development. BMC. Dev. Biol. (2015). https://www.ncbi.nlm.nih.gov/pmc/articles/PMC4506574/

40. Sinha P, Singh K, Sachan M. High resolution methylation analysis of the HOXA5 regulatory region in different somatic tissues of laboratory mouse during development. Gene. Expr. Patterns 23-24 (2017). 59-69

41. Morgan BA. HOX genes and embryonic development. Poult. Sci. 76(1), 96-104 (1997).

42. Nobeyama Y, Umezawa Y, Nakagawa H. Silencing of homeobox A5 gene in the stratum corneum of psoriasis. Exp. Dermatol. 26(11), 1068-1074 (2017).

-• Allows to learn that there were differences in methylation and expression of HOXA5 gene in the psoriatic stage of epidermal maturation.

43. Li B, Tsoi LC, Swindell WR et al. Transcriptome analysis of psoriasis in a large case-control sample: RNA-seq provides insights into disease mechanisms. J. Invest. Dermatol. 134(7), 1828-1838 (2014).

44. Peng X, Zha L, Chen A, Wang Z. HOXA5 is a tumor suppressor gene that is decreased in gastric cancer. Oncol. Rep. 40(3), 1317-1329 (2018).

45. Mo JS, Park YR, Chae SC. Microrna 196b regulates HOXA5, HOXB6 AND GLTP expression levels in colorectal cancer cells. Pathol. Oncol. Res. 25(3), 953-959 (2019).

46. Teo WW, Merino VF, Cho S et al. HOXA5 determines cell fate transition and impedes tumor initiation and progression in breast cancer through regulation of e-cadherin and CD24. Oncogene 35(42), 5539-5551 (2016).

47. Lee DH, Forscher C, Di Vizio D, Koeffler HP. Induction of p53-independent apoptosis by ectopic expression of HOXA5 in human liposarcomas. Sci. Rep. 5, 12580 (2015).

48. Strathdee G, Holyoake TL, Sim A et al. Inactivation of HOXA genes by hypermethylation in myeloid and lymphoid malignancy is frequent and associated with poor prognosis. Clin. Cancer Res. 13(17), 5048-5055 (2007).

49. Liang Y, Xia L, Du Z et al. HOXA5 inhibits keratinocytes growth and epidermal formation in organotypic cultures in vitro and in vivo. J. Dermatol. Sci. 66(3), 197-206 (2012).

50. Lai NS, Wang TF, Wang SL et al. Phostensin caps to the pointed end of actin filaments and modulates actin dynamics. Biochem. Biophys. Res. Commun. 387(4), 676-681 (2009).

51. Kao SC, Chen CY, Wang SL et al. Identification of phostensin, a PP1 F-actin cytoskeleton targeting subunit. Biochem. Biophys. Res. Commun. 356(3), 594-598 (2007).

52. Su YA, Yang J, Tao L, Nguyen H, He P. Undetectable and decreased expression of KIAA1949 (phostensin) encoded on chromosome 6 21.33 in human breast cancers revealed by transcriptome analysis. J. Cancer 1, 38-50 (2010). 